\title{
Peano differentiable extensions in o-minimal structures
}

\author{
Andreas Fischer* \\ (Communicated by T. Grundhöfer)
}

\begin{abstract}
Peano differentiability generalizes ordinary differentiability to higher order. There are two ways to define Peano differentiability for functions defined on non-open sets. For both concepts, we investigate the question under which conditions a function defined on a closed set can be extended to a Peano differentiable function on the ambient space if the sets and functions are definable in an o-minimal structure expanding a real closed field.
\end{abstract}

Key words. o-minimal structure, Peano derivative, extensions.

2000 Mathematics Subject Classification. Primary 03C64; Secondary 14P99, 26B05, 46G05

\section{Introduction}

1.1. The usual notion of differentiability, which we also call Fréchet differentiability, generalizes to higher order in two ways. Let $m$ be a positive integer.

Firstly, we say that a function $f$ is $m$ times Fréchet differentiable if $f$ is Fréchet differentiable, and all partial derivatives of $f$ are $m-1$ times Fréchet differentiable.

Secondly, we stipulate that the function satisfies the Taylor formula of order $m$ at every point, cf. [18]. Let us make this notion, called Peano differentiability, precise.

Suppose $U$ is an open subset of $\mathbb{R}^{n}$. We say that the function $f: U \rightarrow \mathbb{R}$ is $m$ times Peano differentiable at $u \in U$, if there exists an approximation polynomial $p$ of degree $m$ with $p(0)=0$, such that

$$
f(x)-f(u)=p(x-u)+o\left(\|x-u\|^{m}\right) \text { as } x \rightarrow u .
$$

The function $f$ is called $m$ times Peano differentiable if $f$ is $m$ times Peano differentiable at every point of the domain.

Every $m$ times continuously differentiable function is $m$ times Fréchet differentiable, and every $m$ times Fréchet differentiable function is $m$ times Peano differentiable. The approximation polynomial at some point is uniquely determined by the function, so that

${ }^{*}$ Research partially supported by NSERC Discovery Grant of Dr. Salma Kuhlmann and by EC-IHP-Network RAAG (Contract-No: HPRN-CT-2001-00271). 
for an $m$ times continuously differentiable function, both the approximation polynomial and the Taylor polynomial coincide at any point of the domain. For details about the differences of these differentiability concepts, we refer the reader to [13, Example 2.5].

If the domain is closed, we can also define Peano differentiability. Contrary to open domains, the approximation polynomials are not necessarily uniquely determined by $f$, and also Taylor's theorem does not apply anymore, cf. [5]. Therefore, in analogy to continuous differentiability in the sense of Whitney, cf. [27], one either fixes for every point $u$ a certain approximation polynomial; or, one is only interested in the existence of approximation polynomials. In the latter case, we say that $f$ is weakly $m$ times Peano differentiable. In both cases it is natural to ask whether or not such a function is the restriction of an $m$ times Peano differentiable function defined on the ambient space.

1.2. Originating in model theory, o-minimal structures are objects of enormous geometrical potential. Let $R$ be a real closed field. A semialgebraic set is a Boolean combination of sets of the form $\left\{x \in R^{n}: p(x) \geq 0\right\}$, where $p$ is a polynomial in $n$ variables with coefficients of $R$.

An o-minimal expansion $\mathcal{M}$ of $R$ is a sequence of sets $\left(M_{n}\right)_{n \in \mathbb{N}}$ which satisfies the following axioms.

(S1) Each $M_{n}$ is a Boolean algebra of subsets of $R^{n}$, which are called definable.

(S2) Every semialgebraic subset of $R^{n}$ is definable.

(S3) If $A$ and $B$ are definable, then $A \times B$ is definable.

(S4) Projections of definable sets are definable.

(O) The definable subsets of $R$ precisely the finite unions of intervals and points.

A function $f$ is called definable if the graph $\Gamma(f)$ is definable.

A sound introduction to o-minimality is the book [21], and [23] is an excellent survey from the geometrical perspective.

The collection of all semialgebraic sets forms an o-minimal expansion of $R$, cf. [3, Chapter 2]. On the real field, the globally subanalytic sets form the o-minimal structure $\mathbb{R}_{\mathrm{an}}$. In this structure, all analytic functions restricted to compact cubes are definable. The real exponential field $\mathbb{R}_{\text {exp }}$, which consists of the smallest collection $\left(M_{n}\right)_{n \in \mathbb{N}}$ satisfying axioms (S1)-(S4) such that the graph of the entire exponential function is definable, forms also an o-minimal structure, cf. [28, p. 398]. The generation of o-minimal structures is an active research topic, and recent examples of o-minimal structures are constructed in $[22,24,25,16,19]$.

1.3. For each natural number $n$ we endow $R^{n}$ with the Euclidean topology and the Euclidean norm $\|\cdot\|$. For a multi-index $\alpha \in \mathbb{N}^{n}$, let $\alpha !:=\alpha_{1} ! \ldots \alpha_{n} !$ and $|\alpha|:=$ $\alpha_{1}+\cdots+\alpha_{n}$, and, if $x=\left(x_{1}, \ldots, x_{n}\right)$, let $x^{\alpha}:=x_{1}^{\alpha_{1}} \cdots x_{n}^{\alpha_{n}}$.

Let $X$ be a subset of $R^{n}$. A function $f=f_{(0, \ldots, 0)}: X \rightarrow R$ together with the functions $f_{\alpha}: X \rightarrow R$, where $1 \leq|\alpha| \leq m$, is called $m$ times Peano differentiable if for all $x \in X$

$$
f(y)-f(x)=\sum_{1 \leq|\alpha| \leq m} \frac{f_{\alpha}(x)}{\alpha !}(y-x)^{\alpha}+o\left(\|y-x\|^{m}\right) \text { as } y \rightarrow x .
$$


We also say that $\left(f_{\alpha}\right)_{|\alpha| \leq m}$ is $m$ times Peano differentiable relative to $X$, and simply prefix the word definable if all functions $f_{\alpha}$ are definable. The functions $f_{\alpha}$ are called Peano derivatives. If $f$ is a definable $m$ times continuously differentiable function defined on an open set, then, by Taylor's theorem, the functions $f_{\alpha}$ coincide with the usual $\alpha$-th derivative $D_{\alpha} f$.

If for each $|\alpha| \leq m-1$, the function $\left(f_{\alpha+\beta}\right)_{|\beta| \leq m-|\alpha|}$ is additionally $m-|\alpha|$ times Peano differentiable, we say that $f$ is $m$ times Fréchet differentiable.

Moreover, we abbreviate $m$ times Peano, Fréchet and continuously differentiable by the symbols $\mathscr{P}^{m}, \mathscr{F}^{m}$ and $\mathscr{C}^{m}$, respectively.

In this paper, we describe functions defined on closed sets, which can be extended as $m$ times Peano differentiable functions, under the additional assumption that the sets and functions are definable in an o-minimal expansion of a real closed field.

1.4 Results. In general, let $m \geq 2$. The extension problem for Peano differentiable functions defined on closed subsets of $\mathbb{R}$ has been soundly studied in various papers, cf. $[4,17,26]$. The most general result on functions extendable as $m$ times Peano differentiable functions is given by the following theorem, cf. [9], generalizing the corresponding result in [1] for Fréchet differentiable functions, and the one-dimensional case for Peano differentiable functions, cf. [8]. Note that a function $f$ is called a Baire-1 function, if $f$ is the pointwise limit of a sequence of continuous functions.

Theorem 1.1. Let $X \subset \mathbb{R}^{n}$ be closed and let $f_{(0, \ldots, 0)}: X \rightarrow \mathbb{R}$ together with the functions $f_{\alpha}: X \rightarrow \mathbb{R}$, where $1 \leq|\alpha| \leq m$, be $m$ times Fréchet differentiable, such that each $f_{\alpha}$ is a Baire- 1 function for $|\alpha|=m$. Then there is a $\mathscr{P}^{m}$ function $F: \mathbb{R}^{n} \rightarrow \mathbb{R}$ such that $F_{\alpha}=f_{\alpha}$ on $X$ for all $|\alpha| \leq m$.

In the present paper, we study an o-minimal version of Theorem 1.1. That is, we assume that the sets and functions are definable in an o-minimal expansion of a real closed field.

We fix a real closed field $R$ and an o-minimal expansion $\mathcal{M}$ of $R$. The main result is the following theorem, which generalizes [11, Theorem 1.3] for definable Fréchet derivatives.

Theorem 1.2. ${ }^{1}$ Let $A \subset R^{n}$ be a closed definable set. Let $\left(f_{\alpha}\right)_{|\alpha| \leq m}: A \rightarrow R$ be definably $m$ times Peano differentiable relative to $A$. If there exists a finite partition of $A$ into definable sets $A_{1}, \ldots, A_{r}$ such that for every $i=1, \ldots, r$

$$
\left(f_{\alpha}\right)_{|\alpha| \leq m} \text { is } m \text { times Fréchet differentiable in } A_{i},
$$

then there is a definable $m$ times Peano differentiable function $F: R^{n} \rightarrow R$ with

$$
F_{\alpha}=f_{\alpha} \text { on A for all }|\alpha| \leq m .
$$

\footnotetext{
${ }^{1}$ This theorem is from the $\mathrm{PhD}$ thesis of the author
} 
For $n=2$, condition $(*)$ is even necessary. We point out that the sets $A_{i}$ need not to be closed. Moreover, the Baire-1 property is missing in the o-minimal case, as by [11, Theorem 1.5] every definable function satisfies the stronger definable Baire-1 property. That is, every definable function is the pointwise limit of a definable family of continuous functions.

We also study the problem of gluing Peano differentiable functions with closed domains together. This requires the following stronger equality concept.

Let $f, g: R^{n} \rightarrow R$ be functions, and let $A$ be a subset of $R^{n}$. We say that $f$ and $g$ are $\mathscr{P}^{m}$ equal in $A$ if for all $a \in A$

$$
f(x)-g(x) \text { is } o\left(\|x-a\|^{m}\right) \text { as } x \rightarrow a .
$$

If $A$ is an open set, then this is equivalent to the usual equality of functions. For the following theorem, we also allow $m=1$.

Theorem 1.3. Let $A_{1}, \ldots, A_{r} \subset R^{n}$ be definable closed sets, and let $f_{1}, \ldots, f_{r}: R^{n} \rightarrow$ $R$ be $m$ times Peano differentiable functions, such that for $1 \leq i<j \leq r$

$$
f_{i} \text { and } f_{j} \text { are } \mathscr{P}^{m} \text { equal in } A_{i} \cap A_{j} \text {. }
$$

Then there exists an $m$ times Peano differentiable function $F: R^{n} \rightarrow R$ such that for all $j=1, \ldots, r$

$$
F \text { and } f_{j} \text { are } \mathscr{P}^{m} \text { equal in } A_{j} \text {. }
$$

If the functions $f_{1}, \ldots, f_{r}$ are definable, we may choose $F$ to be definable.

This gluing property does not apply to continuously differentiable functions, see [15, Theorem 5.5f] or [20, Proposition 4.7f]. We do not know whether there exist corresponding studies for Peano differentiable functions in classical analysis.

Last we study the extension problem for weakly Peano differentiable functions for functions defined on definable subsets of dimension 1, definable $\mathscr{P}^{m}$ manifolds, and on closed definable subsets of $R^{2}$. For subsets of $R^{2}$ we can give a complete answer in form of the following theorem.

Theorem 1.4. Let $A \subset R^{2}$ be a closed definable subset. Let $f: A \rightarrow R$ be weakly $m$ times Peano differentiable. Then there is an $m$ times Peano differentiable function $F: R^{2} \rightarrow R$ such that $F=f$ on $A$. If $f$ is definable, we may choose $F$ to be definable.

The previous theorem holds in arbitrary dimension and for definable Fréchet differentiable functions ( $m=1)$, cf. [11, Theorem 1.4].

There are 5 sections subsequent to the introduction. In Section 2 we discuss examples concerning the extendability of definable Peano derivatives. In Section 3 and 4 we prove Theorem 1.2 and Theorem 1.3, respectively. In Section 5 we discuss several special cases for the extendability of weakly Peano differentiable functions, and we prove Theorem 1.4. In Section 6 we formulate open questions about extending definable Peano derivatives. 


\section{Examples}

For a subset $S$ of $R^{n}$ let $\chi_{S}: R^{n} \rightarrow R$ denote the characteristic function of $S$.

Next we give examples that show that, in general, we cannot extend $m$ times Fréchet differentiable functions defined on closed sets to $R^{n}$ to $R^{n}$ to $m$ times Fréchet differentiable functions.

Example 2.1. Consider the definable sets $B:=\left\{(x, y): x \geq 0 \wedge y \geq x^{m+1}\right\}$ and $C:=\{(x, y): x \leq 0 \vee y \leq 0\}$. Then $A:=B \cup C$ is definable and closed. Define $\left(f_{\alpha}\right)_{|\alpha| \leq m}: A \rightarrow R$ by

$$
f_{\alpha}(x, y):= \begin{cases}\frac{(m+1) !}{\left(m+1-\alpha_{1}\right) !} x^{m+1-\alpha_{1}} \chi_{B}(x, y), & \text { if } \alpha_{2}=0 \\ 0, & \text { if } \alpha_{2}>0 .\end{cases}
$$

Then $\left(f_{\alpha}\right)_{|\alpha| \leq m}$ is $m$ times Fréchet differentiable relative to $A$, but there is not even a definable $\mathscr{C}^{1}$ function $F: R^{2} \rightarrow R$ with $F=f_{(0,0)}$ on $A$.

Proof. Let $F: R^{2} \rightarrow R$ be a definable Fréchet differentiable function that coincides with $f_{(0,0)}$ in $A$. For $x \geq 0$ we note that

$$
F\left(x, x^{m+1}\right)-F(x, 0)=f\left(x, x^{m+1}\right)-f(x, 0)=x^{m+1} .
$$

For $x>0$, consider the map

$$
y \mapsto F(x, y) .
$$

As $F$ is Fréchet differentiable, we obtain by the Mean Value Theorem a number $y_{x}$ between 0 and $x^{m+1}$ such that

$$
F\left(x, x^{m+1}\right)-F(x, 0)=\frac{\partial F}{\partial y}\left(x, y_{x}\right)\left(x^{m+1}-0\right) .
$$

Therefore,

$$
\frac{\partial F}{\partial y}\left(x, y_{x}\right)=\frac{1}{x}
$$

which implies that the partial derivative of $F$ with respect to $y$ is not bounded at the origin, and thus it is not continuous.

Remark 2.2. If we take $R=\mathbb{R}$ in the previous example, we may conclude that there is not even a (non-definable) $\mathscr{C}^{1}$ function extending $f_{(0,0)}$ to $\mathbb{R}^{2}$.

Remark 2.3. Example 2.1 provides us with explicit functions that show that gluing of $m$ times Fréchet differentiable functions $(m \geq 2)$ without further conditions on the underlying sets does not preserve the differentiability class. The function $f$ restricted to both $B$ and $C$ can be considered as restrictions of polynomials to $B$ and $C$, respectively, and they are $\mathscr{P}^{m}$ equal in $B \cap C$. But there is no $m$ times Fréchet differentiable function $F: R^{2} \rightarrow R$ satisfying $F=f$ on $B$ and $F=f$ on $C$. 
The next example shows that the condition $(*)$ of Theorem 1.2 cannot be dropped without substitution. We recall the following fact. Let $f: U \rightarrow R$ be a definable $\mathscr{P}^{m}$ function, where $U$ is an open subset of $R^{n}$. Then the set $V$, which consists of the points at which $f$ is not $\mathscr{C}^{m}$ smooth, is definable, and

$$
\operatorname{dim}(V) \leq n-2,
$$

cf. [13]. In particular, if $n=2$, then $V$ is finite.

Example 2.4. Let $A:=\{0\} \times R$. Let $\left(f_{\alpha}\right)_{|\alpha| \leq 2}: A \rightarrow R$ be defined by

$$
f_{\alpha}(x, y):= \begin{cases}0, & \text { if } \alpha \neq(1,1), \\ 1, & \text { if } \alpha=(1,1) .\end{cases}
$$

Then $\left(f_{\alpha}\right)_{|\alpha| \leq 2}$ is $m$ times Peano differentiable relative to $A$, but there is no definable $\mathscr{P}^{2}$ function $F: R^{2} \rightarrow R$ with

$$
F_{\alpha}=f_{\alpha} \text { on } A \text { for all }|\alpha| \leq 2 .
$$

Proof. We assume that $f$ is the restriction of a definable 2 times Peano differentiable function $F: R^{2} \rightarrow R$. In this case, the set of points at which $F$ is not $\mathscr{C}^{2}$ smooth is finite. In particular, the function $f_{(1,0)}$ is continuously differentiable with respect to the second variable outside of a finite set. This implies that

$$
\frac{\partial f_{(1,0)}}{\partial y}(0, y)=f_{(1,1)}(0, y)=1
$$

except for finitely many $y \in R$. Obviously, this is not the case.

\section{Proof of Theorem 1.2}

3.1 Preliminary lemmas. In the sequel, we need Escribano's Approximation Theorem, cf. [7, Theorem 1.1].

Theorem 3.1. Let $X \subset R^{n}$ be a definable open set, let $f: X \rightarrow R$ be a definable $\mathscr{C}^{m}$ function, and let $\varepsilon: X \rightarrow(0, \infty)$ be a definable continuous function. Then there is a definable $\mathscr{C}^{m+k}$ function $g: X \rightarrow R$ such that for $x \in X$ and $|\alpha| \leq m$

$$
\left|f_{\alpha}(x)-g_{\alpha}(x)\right|<\varepsilon(x) .
$$

We now solve the extension problem for functions defined on special sets. A definable function $f: X \rightarrow R$ is called a (definable) $\mathscr{C}^{m}$ function, if there exists a definable open neighborhood $U$ of $X$ and a $\mathscr{C}^{m}$ function $F: U \rightarrow R$ such that $\left.F\right|_{X}=f$. For an integer $0 \leq d<n$, we set

$$
X_{0}:=\{(x, 0, \ldots, 0): x \in X\},
$$

which is a subset of $R^{n}$. We use $\bar{A}, A^{\circ}$ and $\partial A$ to denote the closure, interior and frontier of a set $A$, respectively. 
Lemma 3.2. Let $d<n$ and $X \subset R^{d}$ be a definable open set. Let $\left(f_{\alpha}\right)_{|\alpha| \leq m}: \overline{X_{0}} \rightarrow R$ be an definable $m$ times Fréchet differentiable function, such that for all $\alpha \in \mathbb{N}^{n}$ with $|\alpha| \leq m$

(i) $f_{\alpha}$ is $\mathscr{C}^{m}$ smooth in $X_{0}$,

(ii) $f_{\alpha}=0$ on $\partial X_{0}$.

Then, for every definable open neighborhood $U$ of $X_{0}$, there exists a definable $\mathscr{P}^{m}$ function $F: R^{n} \rightarrow R$ such that

(a) $F$ is $\mathscr{C}^{3 m}$ smooth outside of $\overline{X_{0}}$,

(b) $\operatorname{supp}(F) \subset \bar{U}$,

(c) $F_{\alpha}=f_{\alpha}$ on $\overline{X_{0}}$ for $|\alpha| \leq m$.

Proof. Step 1: We define the function $h: X \times R^{n-d} \rightarrow R$ by

$$
h(x, y):=\sum_{\substack{|\alpha| \leq m \\ a_{1}+\cdots+a_{d}=0}} \frac{f_{\alpha}(x, 0)}{\alpha !} y^{\alpha}
$$

for $x \in X$ and $y \in R^{n-d}$. According to property (i), the function $h$ is definable and $m$ times continuously differentiable. Moreover, for $|\alpha| \leq m$,

$$
D_{\alpha} h=f_{\alpha} \text { on } X_{0} .
$$

The function $h$ is not necessarily $\mathscr{C}^{3 m}$ smooth. Let $\varepsilon: X \times R^{n-d} \rightarrow(0, \infty)$ be the definable continuous function given by

$$
\varepsilon(x, y):=\frac{y^{2 m}}{1+y^{2 m}} \operatorname{dist}(x, \partial X), \quad(x, y) \in X \times R^{n-d} .
$$

We apply Theorem 3.1 to $h$ and $\varepsilon$ in place of $f$ and $\varepsilon$, and we obtain a definable $\mathscr{C}^{3 m}$ function $g: X \times R^{n-d} \backslash X_{0} \rightarrow R$ such that for all $|\alpha| \leq m$ and $(x, y) \in X \times\left(R^{n-d} \backslash\{0\}\right)$,

$$
\left|g_{\alpha}(x, y)-h_{\alpha}(x, y)\right|<\varepsilon(x, y) .
$$

Note that for each $\xi \in X_{0}$

$$
\varepsilon(\eta) \text { is } o\left(\|\eta-\xi\|^{m}\right) \text { as } \eta \rightarrow \xi,
$$

so that

$$
|h(\eta)-g(\eta)| \text { is } o\left(\|\eta-\xi\|^{m}\right) \text { as } \eta \rightarrow \xi .
$$

Let $G: X \times R^{n-d}$ denote the unique continuous extension of $g$ to $X \times R^{n-d}$, which is definable. Then $G$ is $m$ times Peano differentiable, and, by the choice of $h$ and $\varepsilon$,

$$
G_{\alpha}=f_{\alpha} \text { on } X_{0}
$$

for all multi-indices $\alpha$ with $|\alpha| \leq m$. 
Step 2: Let $\phi: X \rightarrow(0, \infty)$ be a definable $\mathscr{C}^{3 m}$ function, which satisfies for all $x \in X$

$$
\begin{aligned}
& \phi(x)<\min \{1, \operatorname{dist}((x, 0), \partial U)\}, \\
& \phi(x)<\frac{\operatorname{dist}(x, \partial X)^{m+1}}{1+\|x\|+\max \left\{\left|G_{\alpha}(x)\right|:|\alpha| \leq m\right\}} .
\end{aligned}
$$

Furthermore, let $\rho: R \rightarrow[0,1]$ be a definable $\mathscr{C}^{3 m}$ function that satisfies $\rho(t)=1$ for $|t| \leq 1 / 2$, and $\rho(t)=0$ for $|t| \geq 1$.

We define the function $F: R^{n} \rightarrow R$ by

$$
F(x, y):= \begin{cases}\rho\left(\frac{y}{\phi(x)}\right) G(x, y), & \text { if } x \in X \\ 0, & \text { otherwise }\end{cases}
$$

Step 3: The support of $F$ is evidently contained in $\bar{U}$. The function $F$ is $\mathscr{C}^{3 m}$ smooth in $X \times R^{n-d} \backslash X_{0}$ as well as in

$$
R^{n} \backslash \overline{\left\{(x, y): x \in X, y \in R^{n-d},\|y\|<\phi(x)\right\}},
$$

so that $F$ is $\mathscr{C}^{3 m}$ smooth in $R^{n} \backslash \overline{X_{0}}$. Moreover, the function $F$ is $m$ times Peano differentiable in $R^{n} \backslash \partial X_{0}$. It remains to prove the Peano differentiability at every point of $\partial X_{0}$.

Let $(x, y) \in X \times R^{n-d}$ with $\|y\|<\phi(x)$. Then

$$
\begin{aligned}
|F(x, y)| \leq|G(x, y)| & \leq \sum_{\substack{|\alpha| \leq m \\
a_{1}+\cdots+a_{d}=0}}\left|\frac{f_{\alpha}(x, 0)}{\alpha !} y^{\alpha}\right|+\varepsilon(x, y) \\
& \leq n^{m} \operatorname{dist}(x, \partial X)^{m+1}+\operatorname{dist}(x, \partial X)^{m+1} .
\end{aligned}
$$

Hence, $F(\eta)$ is $o\left(\|\eta-\xi\|^{m}\right)$ as $\eta \rightarrow \xi$ for every $\xi \in \partial X_{0}$.

Finally, the functions $F_{\alpha}$ and $f_{\alpha}$ coincide on $\overline{X_{0}}$ for $|\alpha| \leq m$.

Next, we generalize the previous lemma to functions defined on sets which are graphs of definable Lipschitz continuous $\mathscr{C}^{m}$ functions. This requires the notion of Peano differentiable functions whose domain is $R^{k}$ for some $k$. By $\pi_{i}: R^{k} \rightarrow R$ we denote the projection onto the $i$-th coordinate. For $|\alpha| \leq m$ let $f_{\alpha}: X \rightarrow R^{k}$ be a definable function. We say that $\left(f_{\alpha}\right)_{|\alpha| \leq m}$ is $m$ times Peano differentiable if $\left(\pi_{i} \circ f_{\alpha}\right)_{|\alpha| \leq m}$ is $m$ times Peano differentiable for every $i=1, \ldots, k$. We further set

$$
p_{f, x}(y):=\sum_{1 \leq|\alpha| \leq m} \frac{f_{\alpha}(x)}{\alpha !}(y-x)^{\alpha} .
$$

Remark 3.3. Let $\left(f_{\alpha}\right)_{|\alpha| \leq m}: X \rightarrow Y$ and $\left(g_{\alpha}\right)_{|\alpha| \leq m}: Y \rightarrow Z$ be definable $m$ times Peano differentiable functions. Then their composition $\left(h_{\alpha}\right)_{|\alpha| \leq m}: X \rightarrow Z$ is also $m$ times Peano differentiable. In particular, $h_{(0, \ldots, 0)}:=g_{(0, \ldots, 0)} \circ f_{(0, \ldots, 0)}$ and each $h_{\alpha}(x)$ is 
given as the $\alpha$-th coefficient of the polynomial $p_{g, f(x)} \circ p_{f, x}(y)$ divided by $\alpha$ !. Note, that the functions $h_{\alpha}$ are polynomials in the variables $g_{\beta} \circ f$ and $f_{\beta}$ where $|\beta| \leq m$. If $X$ or $Y$ are not open sets, this definition of the $h_{\alpha}$ is chosen to be compatible with the case of open sets.

The generalization of Lemma 3.2 reads as follows.

Lemma 3.4. Let $X \subset R^{d}$ be a definable open set, and let $h: X \rightarrow R^{n-d}$ be a definable Lipschitz continuous $\mathscr{C}^{2 m}$ function. Set $Y:=\Gamma(h)$. Let $\left(g_{\alpha}\right)_{|\alpha| \leq m}: \bar{Y} \rightarrow R$ be a definable $m$ times Fréchet differentiable function, such that for $|\alpha| \leq m$

(i) $g_{\alpha}$ is $\mathscr{C}^{m}$ smooth in $Y$,

(ii) $g_{\alpha}=0$ on $\partial Y$.

Then for every definable open neighborhood $V$ of $Y$, there is a definable $m$ times Peano differentiable function $G: R^{n} \rightarrow R$ such that

(a) $G$ is $\mathscr{C}^{2 m}$ smooth outside of $\bar{Y}$,

(b) $\operatorname{supp}(G) \subset \bar{V}$,

(c) $G_{\alpha}=g_{\alpha}$ on $\bar{Y}$ for all $|\alpha| \leq m$.

Proof. Step 1: Let $\psi: \bar{X} \times R^{n-d} \rightarrow \bar{X} \times R^{n-d}$ be the function defined by

$$
\psi(x, y):=(x, y+h(x)) .
$$

Then the function $\psi$ is $\mathscr{C}^{2 m}$ smooth in $X \times R^{n-d}$, and it is Lipschitz continuous with Lipschitz continuous inverse on $\bar{X} \times R^{n-d}$. Let $L$ be a Lipschitz constant that is valid for both functions $\psi$ and $\psi^{-1}$.

Step 2: We define the functions $\left(f_{\alpha}\right)_{|\alpha| \leq m}$ as follows. On $X_{0}$, we define the functions $f_{\alpha}$ according to Remark 3.3 with $f, g$ and $\psi$ in place of $h, g$ and $f$. Therefore, the $f_{\alpha}$ are $\mathscr{C}^{m}$ functions on $X_{0}$. For any $\eta \in \partial Y$, property (ii) implies that

$$
g(\zeta) \text { is } o\left(\|\zeta-\eta\|^{m}\right) \text { as } \zeta \rightarrow \eta \text {. }
$$

Hence, for every $\xi \in \partial X_{0}$,

$$
f(\zeta)=g(\psi(\zeta)) \text { is } o\left(\|\psi(\zeta)-\psi(\xi)\|^{m}\right) \text { as } \zeta \rightarrow \xi,
$$

so that the Lipschitz continuity of $\psi$ implies that

$$
f(\zeta) \text { is } o\left(\|\zeta-\xi\|^{m}\right) \text { as } \zeta \rightarrow \xi .
$$

Thus, if we set $f_{\alpha}(\xi):=0$ for $\xi \in \partial X_{0}$, the function $f_{(0, \ldots, 0)}$ together with the functions $f_{\alpha}$, where $1 \leq|\alpha| \leq m$, is $m$ times Peano differentiable and satisfies the conditions of Lemma 3.2.

Step 3: Let $U$ be the intersection of the sets

$$
\left\{x: \operatorname{dist}\left(x, X_{0}\right)<\operatorname{dist}\left(x, \partial X_{0}\right)\right\} \text { and } \psi^{-1}(V) .
$$


Then, by Lemma 3.2, there is a definable $\mathscr{P}^{m}$ function $F: R^{n} \rightarrow R$ that satisfies the conclusions of Lemma 3.2. We claim that $G: R^{n} \rightarrow R$,

$$
G(u):= \begin{cases}F\left(\psi^{-1}(u)\right), & \text { if } u \in \psi(U), \\ 0, & \text { otherwise, }\end{cases}
$$

suits the desired properties.

Property (a) is evidently satisfied, and $G$ is $\mathscr{C}^{2 m}$ smooth outside of $\overline{\psi(U)}$. Moreover, the function $F$ is $\mathscr{P}^{m}$ smooth in $R^{n} \backslash \partial Y$, and, by construction,

$$
F_{\alpha}=f_{\alpha} \text { in } Y \text { for }|\alpha| \leq m .
$$

In order to verify that for every $\eta \in \partial Y$

$$
F(\zeta) \text { is } o\left(\|\zeta-\eta\|^{m}\right) \text { as } \zeta \rightarrow \eta,
$$

we note that for any $\xi \in \partial X_{0}$,

$$
G(\zeta) \text { is } o\left(\|\zeta-\xi\|^{m}\right) \text { as } \zeta \rightarrow \xi .
$$

So

$$
F(\zeta)=G\left(\psi^{-1}(\zeta)\right) \text { is } o\left(\left\|\psi^{-1}(\zeta)-\psi^{-1}(\eta)\right\|^{m}\right) \text { as } \zeta \rightarrow \eta,
$$

and hence, by the Lipschitz continuity of $\psi^{-1}$, equation (3.1) is evident.

3.2. We prove Theorem 1.2. This requires a special kind of partition of definable sets. In the following theorem we summarize several decomposition concepts in o-minimal geometry, cf. [21, p. 115f] and [12, Theorem 1.4].

Theorem 3.5. Let $k>0$ be an integer. Let $A:=A_{1} \cup \cdots \cup A_{r} \subset R^{n}$ be a union of definable sets, and let $f: A \rightarrow R^{k}$ be definable. There exists a finite partition of $A$ into definable $\mathscr{C}^{m}$ sub-manifolds $B_{1}, \ldots, B_{s}$, called strata, such that

(a) each $A_{i}$ is the union of some of the strata,

(b) each stratum $B_{j}$ is either open, or, after some linear orthogonal change of coordinates, $B_{j}=\Gamma\left(h_{j}\right)$, where $h_{j}$ is a Lipschitz continuous $\mathscr{C}^{k}$ function with open domain, $j=1, \ldots, s$,

(c) $f \mid B_{j}$ is a definable $\mathscr{C}^{k}$ function for all $j=1, \ldots, s$,

(d) every $B_{j}$ has a definable open neighborhood $V_{j}$ which is disjoint to $B_{\ell}$ for $\ell \neq j$ and $\operatorname{dim}\left(B_{\ell}\right) \leq \operatorname{dim}\left(B_{j}\right)$.

In case of item (a), we also say that the partition is compatible with $A_{1}, \ldots, A_{r}$.

The dimension of a definable set $X$ is the maximal integer $\operatorname{dim}(X)$ such that $X$ contains a set, which is definably homeomorphic to $R^{\operatorname{dim}(X)}$. According to [21, p. 67], every definable set $X$ satisfies the following inequality:

$$
\operatorname{dim}(\partial X)<\operatorname{dim}(X) .
$$

This implies that $\bar{X}$ has the same dimension as $X$ for every definable set $X$. 
Proof of Theorem 1.2. According to Theorem 3.5, we may select a finite partition of $A$ into definable sets $B_{1}, \ldots, B_{s}$ of the form (b) of Theorem 3.5 with $k=2 m$, which is compatible with the sets $A_{1}, \ldots, A_{r}$, such that the function $f_{\alpha}$ restricted to $B_{i}$ is $\mathscr{C}^{m}$ smooth for each $|\alpha| \leq m$ and $i=1, \ldots, s$. By permuting the indices, we may further assume that

$$
\operatorname{dim}\left(B_{i}\right) \leq \operatorname{dim}\left(B_{i+1}\right)
$$

for $i=1, \ldots, s-1$. Hence, for any $t=1, \ldots, s$, the set $\bigcup_{i=1}^{t} B_{i}$ is closed.

We prove by induction on $t$ that there exists a definable $\mathscr{P}^{m}$ function $F_{t}: R^{n} \rightarrow R$, such that $F_{\alpha}=f_{\alpha}$ on $\bigcup_{i=1}^{t} B_{i}$ for all $|\alpha| \leq m$, and such that $F$ is $\mathscr{C}^{2 m}$ smooth outside of $\bigcup_{i=1}^{t} B_{i}$.

The case $t=1$ is evident by Lemma 3.4.

Suppose that we have constructed $F_{t}$. For each $|\alpha| \leq m$ let $g_{\alpha}: \overline{B_{t+1}} \rightarrow R$ be defined by $g_{\alpha}:=f_{\alpha}-\left(F_{t}\right)_{\alpha}$. Then each $g_{\alpha}$ vanishes on $\partial B_{t+1}$, and $g_{\alpha}$ restricted to $B_{t+1}$ is a $\mathscr{C}^{m}$ function as $F_{t}$ is $\mathscr{C}^{2 m}$ smooth outside of $\bigcup_{i=1}^{t} B_{i}$.

Select, by Theorem 3.5 (d), a definable open neighborhood $V$ of $B_{t+1}$, which has empty intersection with $\bigcup_{i=1}^{t} B_{i}$. Then, by Lemma 3.4, there exists a definable $\mathscr{P}^{m}$ function $G: R^{n} \rightarrow R$, which satisfies the conclusions of Lemma 3.4. Therefore, $F_{t+1}:=$ $G+F_{t}$ satisfies the desired properties.

Set $F:=F_{s}$.

3.3 Corollaries and remark. As an immediate consequence of Theorem 1.2 we note the o-minimal version of Theorem 1.1.

Corollary 3.6. Let $A \subset R^{n}$ be closed and definable, and let $\left(f_{\alpha}\right)_{|\alpha| \leq m}: A \rightarrow R$ be a definable $\mathscr{F}^{m}$ function relative to $A$. Then there is a definable $\mathscr{P}^{m}$ function $F: R^{n} \rightarrow$ $R$, such that

$$
F_{\alpha}=f_{\alpha} \text { on A for all }|\alpha| \leq m
$$

Corollary 3.7. Let $A \subset R$ be a definable closed set, and let $\left(f_{\alpha}\right)_{|\alpha| \leq m}: A \rightarrow R$ be definably $m$ times Peano differentiable relative to $A$. Then there is a definable $m \mathscr{P}^{m}$ function $F: R \rightarrow R$ such that $F_{\alpha}=f_{\alpha}$ on Afor $|\alpha| \leq m$.

Proof. By o-minimality, the set $A$ is a finite disjoint union of open intervals and singletons. On an open set, the Peano derivatives are determined by the function itself, and by inequality (2.1), a unary definable Peano differentiable function is continuously differentiable. Thus condition $(*)$ of Theorem 1.2 is satisfied.

Remark 3.8. The condition $(*)$ of Theorem 1.2 is also necessary if $n=2$.

Proof. This follows from the fact that definable $m$ times Peano differentiable functions defined on an open subset of $R^{2}$ are $m$ times continuously differentiable outside of a finite subset. Moreover, every function restricted to a singleton is $m$ times Fréchet differentiable. 


\section{Gluing properties}

4.1 Open sets. If $A$ is an open set and $f$ and $g$ are $m$ times Peano differentiable in $A$, then $f$ and $g$ are $\mathscr{P}^{m}$ equal in $A$ if and only if $g=f$ in $A$.

Remark 4.1. The gluing property of finitely many definable $m$ times Peano differentiable functions, whose domains are open, reads as follows.

Let $U_{1}, \ldots, U_{r}$ be definable open subsets of $R^{n}$, and for $i=1, \ldots, r$ let $f_{i}: U_{i} \rightarrow R$ be definable $m$ times Peano differentiable functions such that $f_{i}=f_{j}$ on $U_{i} \cap U_{j}$ for $1 \leq$ $i, j \leq r$. Then there is a definable $m$ times Peano differentiable function $F: \bigcup_{i} U_{i} \rightarrow R$ such that $F=f_{i}$ on $U_{i}$ for $i=1, \ldots, r$.

The proof is a standard application of definable $\mathscr{C}^{m}$ partition of unity, and we omit it. If $R=\mathbb{R}$, then definability is not needed, and $r=\infty$ is also allowed.

4.2 Closed sets. More difficulties appear if we want to glue definable weakly $m$ times Peano differentiable functions with closed domains. We do not know whether there exist corresponding studies for gluing $m$ times Peano differentiable functions without the ominimality assumption on the sets.

Lemma 4.2. Let $C \subset R^{n}$ be a set of the form of Theorem 3.5 (b), and let $U$ be a definable open neighborhood of $C$. Let $f: R^{n} \rightarrow R$ be an $m$ times Peano differentiable function, which is $\mathscr{P} m$ equal to the zero function in $\partial C$. Then there is an $m$ times Peano differentiable function $F: R^{n} \rightarrow R$, which vanishes outside of $U$ such that $F$ and $f$ are $\mathscr{P}^{m}$ equal in $\bar{C}$. If $f$ is definable, we may choose $F$ to be definable.

Proof. If $C$ is open, then set $F=f$ in $\bar{C}$ and $F=0$ outside of $\bar{C}$. Otherwise, after some suitable change of coordinates, we may assume that $C=\Gamma(h)$ where $X \subset R^{d}$ is an open definable set and $h: X \rightarrow R^{n-d}$ is a definable Lipschitz continuous $\mathscr{C}^{m}$ function. Let $\varphi: R^{d} \rightarrow[0, \infty)$ be a definable $\mathscr{C}^{m}$ function that vanishes outside of $X$, and which satisfies

$$
0<\varphi(x)<\operatorname{dist}\left(h(x), R^{n} \backslash U\right)
$$

for every $x \in X$. Let $\rho: R \rightarrow[0,1]$ be a definable $\mathscr{C}^{m}$ function which equals 1 in $[-1 / 2,1 / 2]$ and vanishes outside of $(-1,1)$. Then the function $F: R^{n} \rightarrow R$,

$$
F(x, y):= \begin{cases}f(x, h(x)) \rho\left(\frac{y-h(x)}{\varphi(x)}\right), & \text { if } x \in X \\ 0, & \text { otherwise }\end{cases}
$$

suits the desired properties. Moreover, as $h, \rho$ and $\varphi$ are definable, the function $F$ is definable if $f$ is definable.

Proof of Theorem 1.3. According to Theorem 3.5, we select a finite partition of $R^{n}$ that is compatible with the sets $A_{1}, \ldots, A_{r}$, and we denote by $B_{1}, \ldots, B_{k}$ the strata which are contained in at least one of the sets $A_{s}$. Without loss of generality we may assume that the strata are ordered in such a way that

$$
\operatorname{dim}\left(B_{i}\right) \leq \operatorname{dim}\left(B_{i+1}\right)
$$


for $i=1, \ldots, k-1$. Note that the set $\bigcup_{i=1}^{\ell} B_{i}$ is closed, and that there is a definable open neighborhood $U_{\ell+1}$ of $B_{\ell+1}$ such that for each $\ell=1, \ldots, k-1$,

$$
\bigcup_{i=1}^{\ell} B_{i} \cap U_{\ell+1}=\emptyset
$$

We prove by induction on $\ell$ that there exists a $\mathscr{P}^{m}$ function $F_{\ell}: R^{n} \rightarrow R$ such that for every $i=1, \ldots, k$ and $s=1, \ldots, r$ the functions $F_{\ell}$ and $f_{i}$ are $\mathscr{P}^{m}$ equal in $A_{s} \cap$ $\bigcup_{j=1}^{\ell} B_{j}$.

The case $\ell=1$ is evident.

Step from $\ell$ to $\ell+1$ : For $i=1, \ldots, k$ let $h_{i}=F_{\ell}-f_{i}$. Then $h_{i}$ and the zero function are $\mathscr{P}^{m}$ equal in $\bigcup_{i=1}^{\ell} B_{i}$. Note that $\partial B_{\ell+1}$ is contained in $\bigcup_{i=1}^{\ell} B_{i}$. By Lemma 4.2 there is a $\mathscr{P}^{m}$ function $g_{\ell}: R^{n} \rightarrow R$ which is $\mathscr{P}^{m}$ equal to $h_{i}$ in $A_{s} \cap \overline{B_{\ell+1}}, s=1, \ldots, r$, and which vanishes outside of $U_{\ell+1}$. Now $F_{\ell+1}=F_{\ell}-g_{\ell}$ satisfies the desired properties.

Set $F=F_{k}$. If the functions $f_{1}, \ldots, f_{r}$ are additionally definable, then, by Lemma 4.2, we may select the functions $F_{i}, h_{i}$ and $g_{i}$ to be definable, so that $F$ is definable.

\section{Weakly $\boldsymbol{m}$ times Peano differentiable functions}

Now we consider another extension problem. First we make weak Peano differentiability precise.

Definition 5.1. A function $f: A \rightarrow R$ is called weakly $m$ times Peano differentiable if there is for every $a \in A$ a polynomial $p \in R[X]$ with $\operatorname{deg}(p) \leq m$ and $p(0)=0$ such that

$$
f(x)-f(a)=p(x-a)+o\left(\|x-a\|^{m}\right) \text { as } x \rightarrow a .
$$

Note that we omit the word weak if the set $A$ is open.

5.1 One-dimensional sets. Our first aim to prove extendability for weakly $m$ times Peano differentiable functions defined on definable closed sets of dimension 1. In this case we can treat both definable and arbitrary functions.

Lemma 5.2. Let $h:(b, c) \rightarrow R^{n-1}$ be a definable Lipschitz continuous $\mathscr{C}^{m}$ mapping, and let $U$ be a definable open neighborhood of $\Gamma(h)$. If $f: \overline{\Gamma(h)} \rightarrow R$ is a weakly $\not P^{m}$ function such that for $a \in \partial \Gamma(h)$,

$$
f(x) \text { is } o\left(\|x-a\|^{m}\right) \text { as } x \rightarrow a,
$$

then there is a $\mathscr{P}^{m}$ function $F: R^{n} \rightarrow R$ which satisfies

(a) $F=f$ on $\overline{\Gamma(h)}$,

(b) $\operatorname{supp}(F) \subset \bar{U}$.

If $f$ is definable we may choose $F$ to be definable. 
Proof. The function $h$ is Lipschitz continuous and definable, so that $h$ extends to $[a, b]$ as Lipschitz continuous function $\bar{h}$ with Lipschitz constant $L>0$. Therefore,

$$
f(t, h(t)) \text { is } o\left(|t-a|^{m}\right) \text { as } t \rightarrow a
$$

for every $a \in \partial(b, c)$. In addition, the map $t \mapsto f(t, h(t))$ is a weakly $\mathscr{P}^{m}$ function on $(b, c)$, so that $t \mapsto f(t, \overline{h(t)})$ is weakly $\mathscr{P}^{m}$ in $[a, b]$. Let $\varphi:(b, c) \rightarrow(0, \infty)$ be a definable $\mathscr{C}^{m}$ function such that the set

$$
V:=\left\{x=\left(x_{1}, \ldots, x_{n}\right):\left\|x-\left(x_{1}, h\left(x_{1}\right)\right)\right\|<\varphi\left(x_{1}\right)\right\}
$$

is contained in $U$, and let $\rho: R \rightarrow[0,1]$ be a definable $\mathscr{C}^{m}$ function which equals 1 in $[-1 / 2,1 / 2]$ and vanishes outside of $(-1,1)$. Then, the function $F: R^{n} \rightarrow R$,

$$
F\left(x_{1}, \ldots, x_{n}\right):= \begin{cases}f\left(x_{1}, h\left(x_{1}\right)\right) \rho\left(\frac{\left\|\left(x_{2}, \ldots, x_{n}\right)-h\left(h_{1}\right)\right\|}{\varphi\left(x_{1}\right)}\right), & \text { if } x_{1} \in(b, c), \\ 0, & \text { otherwise }\end{cases}
$$

suits our needs. Note that the functions $h, \varphi$ and $\rho$ are definable, so that $F$ is definable if $f$ is definable.

The following proposition answers affirmatively the extension property for weakly $\mathscr{P}^{m}$ functions defined on closed definable subsets of dimension 1.

Proposition 5.3. Let $A \subset R^{n}$ be a definable closed set of dimension 1. Then, for every weakly $m$ times Peano differentiable function $f: A \rightarrow R$ there exists a $\mathscr{P}^{m}$ function $F: R^{n} \rightarrow R$ such that

$$
F=f \text { on } A \text {. }
$$

If $f$ is definable, then $F$ can be chosen to be definable.

Proof. We select a finite partition $B_{1}, \ldots, B_{s}$ of $A$ into sets with properties (a), (b), and (d) of Theorem 3.5. Let $B_{1}, \ldots, B_{r}$ denote the singletons, and $B_{r+1}, \ldots, B_{s}$ be the sets of dimension 1.

For $i=1, \ldots, r$, let the polynomial $p_{i}$ be chosen in such a way that

$$
f(x)-f\left(b_{i}\right)=p_{i}\left(x-b_{i}\right)+o\left(\left\|x-b_{i}\right\|^{m}\right) \text { as } x \rightarrow b_{i}
$$

where $B_{i}=\left\{b_{i}\right\}$.

Then the function $G: R^{n} \rightarrow R$ which is defined by

$$
G(x)=\sum_{i=1}^{r} \rho\left(\frac{x-b_{i}}{r}\right)\left(f\left(b_{i}\right)+p_{i}\left(x-b_{i}\right)\right)
$$

is $m$ times Peano differentiable and definable. For each $j=r+1, \ldots, s$, the function $(f-G)$ restricted to $B_{j}$ additionally satisfies, after some suitable change of coordinates, the conditions of Lemma 5.2. 
We select for each $j=r+1, \ldots, s$ a definable open neighborhood $U_{j}$ of $B_{j}$ which has empty intersection with $B_{\ell}$ for $\ell \neq j$. Lemma 5.2 provides $\mathscr{P}^{m}$ functions $g_{j}: R^{n} \rightarrow R$ for $j=r+1, \ldots, s$, such that $\operatorname{supp}\left(g_{j}\right) \subset \overline{U_{j}}$ and $g_{j}(b)=(f-G)(b), b \in \overline{B_{j}}$.

So,

$$
F=G+\sum_{j=r+1}^{s} g_{j}
$$

suits the desired properties.

If $f$ is definable, then the functions $g_{j}$ can be chosen definable by Lemma 5.2, so that $F$ is definable.

5.2 Peano differentiable manifolds. The definition of Peano differentiable manifolds is similar to that of $\mathscr{C}^{m}$ manifolds. Here, all manifolds are embedded. A definable set $S \subset R^{n}$ is called a definable $\mathscr{P}^{m}$ manifold if for every $x \in S$ there exists a definable open neighborhood $U$ of $x$ and a definable $\mathscr{P}^{m}$ diffeomorphism $\Phi$ from $U$ to the unit ball $B_{1}(0)$ such that $\Phi(x)=0$ and $\Phi(U \cap S)=\left\{\left(x_{1}, \ldots, x_{n}\right) \in B_{1}(0): x_{n-d+1}=\right.$ $\left.\cdots=x_{n}=0\right\}$. An atlas of a definable $\mathscr{P}^{m}$ manifold is called definable if all charts are definable.

By [2, 2.4], every definable $\mathscr{C}^{m}$ manifold has a finite definable special atlas (of $\mathscr{C}^{m}$ charts); that is, a finite definable atlas whose charts are linear projections. This is a consequence of the continuity of the tangent mapping of a $\mathscr{C}^{m}$ manifold.

The tangent mapping of $\mathscr{P}^{m}$ manifolds is in general not continuous, and we do not know whether or not every definable $\mathscr{P}^{m}$ manifold possesses a finite definable atlas. Thus we restrict ourselves to manifolds with finite definable special atlases.

Proposition 5.4. Let $A \subset R^{n}$ be a closed definable $\mathscr{P}^{m}$ manifold, which possesses a finite definable special atlas. Let $f: A \rightarrow R$ be a weakly $\mathscr{P}^{m}$ function. Then there is a $\mathscr{P}^{m}$ function $F: R^{n} \rightarrow R$ such that $F=f$ on $A$. If $F$ is definable, we can choose $F$ to be definable.

Proof. Let $\phi_{i}: S_{i} \rightarrow U_{i} \subset R^{d_{i}}$ be the charts of the atlas, $i=1, \ldots, r$. Then each $\phi_{i}$ extends to a definable $\mathscr{P}^{m}$ diffeomorphism $\Phi_{i}$ from a an open definable neighborhood of $S_{i}$ in $R^{n}$ to an open definable neighborhood $W_{i}$ of $U_{i} \times\{0\}$ in $U_{i} \times R^{n-d_{i}}$. We may additionally assume that if $(u, y) \in W_{i}$, then the segment connecting $(u, 0)$ and $(u, y)$ is contained in $W_{i}$. For each $i$, we extend $f \circ \phi_{i}^{-1}$ to the $\mathscr{P}^{m}$ function $F_{i}$ defined on $W_{i}$ by setting $F_{i}(u, y)=f_{i} \circ \phi_{i}^{-1}(u)$. The sets $U_{1}, \ldots, U_{r}$ cover $A$. Let $U_{0}:=R^{n} \backslash A$. Select a definable $\mathscr{C}^{m}$ partition of unity $\varphi_{0}, \ldots, \varphi_{r}: R^{n} \rightarrow R$ subordinate to the sets $U_{0}, \ldots, U_{r}$. Then the function $F: R^{n} \rightarrow R$ given by

$$
F:=\sum_{i=1}^{r} \varphi_{i} F_{i} \circ \phi_{i}
$$

has the desired properties. As the functions $\varphi_{1}, \ldots, \varphi_{r}$ and $\phi_{1}, \ldots, \phi_{r}$ are definable, the function $F$ is definable if $f$ is definable. 
5.3 Sets in $\boldsymbol{R}^{2}$. We prepare the proof of Theorem 1.4 by the following lemma.

Lemma 5.5. Let $f, g:(a, b) \rightarrow R$ be definable $\mathscr{C}^{m}$ functions with $f(t)<g(t)$ for all $t \in(a, b)$. Let

$$
C:=\{(x, y): x \in(a, b) \wedge f(x)<y \leq g(x)\} .
$$

Let $F: C \rightarrow R$ be an $m$ times weakly Peano differentiable function. Then there exists a definable open neighborhood $U$ of $C$ and an $m$ times Peano differentiable function $G: U \rightarrow R$ such that $G=F$ on $C$.

Proof. By applying the function $\psi:(a, b) \times R \rightarrow(a, b) \times R$ defined by

$$
\psi(x, y):=(x, y-g(x)),
$$

we may assume that $g \equiv 0$. Consider the linear system

$$
\left(\begin{array}{ccccc}
1 & 1 & 1 & \ldots & 1 \\
0 & -1 & -2 & \ldots & -m \\
0 & (-1)^{2} & (-2)^{2} & \ldots & (-m)^{2} \\
\vdots & \vdots & \vdots & & \vdots \\
0 & (-1)^{m} & (-2)^{m} & \ldots & (-m)^{m}
\end{array}\right)\left(\begin{array}{c}
a_{0} \\
a_{1} \\
a_{2} \\
\vdots \\
a_{m}
\end{array}\right)=\left(\begin{array}{c}
1 \\
1 \\
1 \\
\vdots \\
1
\end{array}\right)
$$

The above $(m+1) \times(m+1)$-matrix is of Vandermonde type, whose generators $0,-1, \ldots$, $-m$ are pairwise distinct. Thus this matrix is invertible, and the system (5.1) has a unique solution $a_{0}, \ldots, a_{m}$. We set

$$
U:=\left\{(x, y): x \in(a, b) \wedge f(x)<y<\frac{-f(x)}{m+1}\right\},
$$

and define $G: U \rightarrow R$ by

$$
G(x, y):= \begin{cases}F(x, y), & y<0 \\ \sum_{k=0}^{m} a_{k} F(x,-k y), & y \geq 0\end{cases}
$$

By the choice of $a_{0}, \ldots, a_{n}$, the functions $F$ and $\sum_{k=0}^{m} a_{k} F(x,-k y)$ are $\mathscr{P}^{m}$ equal in $(a, b) \times\{0\}$, so that they glue together to the $\mathscr{P}^{m}$ function $G$.

Note that if $F$ is definable, then also $G$ is definable.

Corollary 5.6. Let $f, g, F, C$ and $U$ be as in Lemma 5.5. If $F$ is $o\left(\|x-b\|^{m}\right)$ as $x \rightarrow b$ for every $b \in \partial \Gamma(g)$, then we may assume that the function $G$ is $\mathscr{P}^{m}$ equal to 0 in $\Gamma(-f /(m+2))$.

Proof. Let $\rho: R \rightarrow[0,1]$ be a definable $\mathscr{C}^{m}$ function that equals 1 for $t \leq 0$ and vanishes for $t \geq 1 / 2$. Then the function $G$ from equation (5.2) multiplied with

$$
\rho\left(\frac{y(m+2)}{f(x)}\right)
$$

has the desired property. 
We are now able to prove Theorem 1.4.

Proof. Consider the sets

$$
\overline{A^{\circ}} \text { and } \overline{A \backslash \overline{A^{\circ}}} \text {. }
$$

The dimension of the latter set is bounded by 1 , and $\overline{A^{\circ}} \cap \overline{A \backslash \overline{A^{\circ}}}$ is a finite set. By arguing as in the proof of Proposition 5.3 we may assume that

$$
f(x) \text { is } o\left(\|x-b\|^{m}\right) \text { as } x \rightarrow b
$$

for every $b \in \overline{A^{\circ}} \cap \overline{A \backslash \overline{A^{\circ}}}$. Hence, by Theorem 1.3 and Proposition 5.3 it remains to prove that $f$ restricted to $\overline{A^{\circ}}$ extends to $R^{2}$, as the extendability of $f$ restricted to $\overline{A \backslash \overline{A^{\circ}}}$ was already proved in Proposition 5.3. Select a finite partition of $\overline{A^{\circ}}$ into definable $\mathscr{C}^{m}$ submanifolds $B_{1}, \ldots, B_{s}$. We may additionally assume that $\operatorname{dim}\left(B_{i}\right) \leq \operatorname{dim}\left(B_{i+1}\right)$ for $i=1, \ldots, s-1$. If $B_{j}=\left\{b_{j}\right\}$ is a singleton contained in the boundary of $\overline{A^{\circ}}$, there is a polynomial $p_{j}$ such that $f$ and $p_{j}$ are $\mathscr{P}^{m}$ equal in $B_{j}$. Select a definable $\mathscr{C}^{m}$ function $\varphi_{j}: R^{2} \rightarrow R$ which equals 1 in a sufficiently small open neighborhood of $b_{j}$, and that vanishes in a definable open neighborhood of the other $B_{\ell}$ with $\operatorname{dim}\left(B_{\ell}\right)=0$. Then

$$
f(x)-\varphi p_{j}\left(x-b_{j}\right) \text { is } o\left(\left\|x-b_{j}\right\|^{m}\right) \text { as } x \rightarrow b_{j} .
$$

So we may assume that

$$
f(x) \text { is } o\left(\left\|x-b_{j}\right\|^{m}\right) \text { as } x \rightarrow b_{j}
$$

for each $j$ with $\operatorname{dim}\left(B_{j}\right)=0$. If $B_{j}$ is contained in the boundary of $\overline{A^{\circ}}$ such that $\operatorname{dim}\left(B_{j}\right)=1$, then there is a definable open neighborhood $U_{j}$ of $B_{j}$ and a $\mathscr{P}^{m}$ function $G_{j}: U_{j} \rightarrow R$ such that $G_{j}$ and $f$ are $\mathscr{P}^{m}$ in $U \cap A$. Moreover, the function $f$ is $\mathscr{P}^{m}$ equal to 0 at every point $b \in \overline{B_{j}} \backslash B_{j}$. We glue the $G_{j}$ and $f \mid \overline{A^{\circ}}$ together to the function $F$. By Corollary 5.6, we can extend this function to $R^{2}$ by setting $F(x)=0$ outside of a closed definable neighbourhood of $\overline{A^{\circ}}$.

Note that if $f$ is definable then the functions $G_{j}$ are definable, and so $F$ is definable.

\section{Open questions}

Consider the Example 2.4 for $R=\mathbb{R}$. We do not know whether $f$ can be extended as (non-definable) 2 times Peano differentiable function or not. It would be interesting whether or not a definable $\mathscr{P}^{m}$ function $f: A \rightarrow \mathbb{R}$ is the restriction of a definable $\mathscr{P}^{m}$ function $F: \mathbb{R}^{n} \rightarrow \mathbb{R}$ if and only if $f$ is the restriction of a (non-definable) $\mathscr{P}^{m}$ function $G: \mathbb{R}^{n} \rightarrow \mathbb{R}$.

Furthermore, the condition $(*)$ of Theorem 1.2 is only known to be necessary in the case $n=2$. So far, no definable $m$ times Peano differentiable function with open domain is known that does not satisfy this condition. The following question arises: Can condition (*) be weakened, or does every definable $\mathscr{P}^{m}$ function with open domain satisfy $(*)$ ?

Finally, can Theorem 1.4 be generalized to higher dimension, or what are the sets that admit extendability? 


\section{References}

[1] V. Aversa, M. Laczkovich, D. Preiss, Extension of differentiable functions. Comment. Math. Univ. Carolin. 26 (1985), 597-609. MR817830 (87c:26022) Zbl 0583.26003

[2] A. Berarducci, M. Otero, Intersection theory for o-minimal manifolds. Ann. Pure Appl. Logic 107 (2001), 87-119. MR1807841 (2001m:03074) Zbl 0968.03044

[3] J. Bochnak, M. Coste, M.-F. Roy, Real algebraic geometry. Springer 1998. MR1659509 (2000a:14067) Zbl 0912.14023

[4] Z. Buczolich, C. E. Weil, Extending Peano differentiable functions. Atti Sem. Mat. Fis. Univ. Modena 44 (1996), 323-330. MR1428765 (98b:26002) Zbl 0865.26007

[5] Z. Buczolich, C. E. Weil, The non-coincidence of ordinary and Peano derivatives. Math. Bohem. 124 (1999), 381-399. MR1722874 (2000i:26005) Zbl 0936.26002

[6] M. Coste, An Introduction to O-minimal Geometry. Dip. Mat. Univ. Pisa, Dottorato di Ricerca in Matematica, Istituti Editoriali e Poligrafici Internazionali, Pisa, 2000.

[7] J. Escribano, Approximation theorems in o-minimal structures. Illinois J. Math. 46 (2002), 111-128. MR1936078 (2003i:03042) Zbl 1010.03026

[8] H. Fejzić, J. Mařík, C. E. Weil, Extending Peano derivatives. Math. Bohem. 119 (1994), $387-$ 406. MR1316592 (96c:26003) Zbl 0824.26003

[9] H. Fejzić, D. Rinne, C. Weil, Extending $n$ times differentiable functions of several variables. Czechoslovak Math. J. 49(124) (1999), 825-830. MR1746707 (2001f:26014) Zbl 1005.26007

[10] A. Fischer, Peano-Differentiable Functions in O-minimal Structures. Doctoral thesis, Universität Passau, 2006.

[11] A. Fischer, Extending o-minimal Fréchet derivatives. Ann. Polon. Math. 92 (2007), 179-189. MR2328887 (2008e:03064) Zbl 1134.03023

[12] A. Fischer, o-minimal $\Lambda^{m}$-regular stratification. Ann. Pure Appl. Logic 147 (2007), 101-112. MR2328201 Zbl 1125.03029

[13] A. Fischer, Singularities of o-minimal Peano derivatives. Math. Nach., accepted.

[14] T. Kaiser, J.-P. Rolin, P. Speissegger, Transition maps at non-resonant hyperbolic singularities are o-minimal. J. Reine Angew. Math., to appear.

[15] B. Malgrange, Ideals of differentiable functions. Tata Institute of Fundamental Research, Bombay 1967. MR0212575 (35 \#3446) Zbl 0177.17902

[16] C. Miller, Expansions of the real field with power functions. Ann. Pure Appl. Logic 68 (1994), 79-94. MR1278550 (95i:03081) Zbl 0823.03018

[17] S. N. Mukhopadhyay, S. Ray, On extending Peano derivatives. Acta Math. Hungar. 89 (2000), 327-346. MR1912764 (2003d:26005) Zbl 0973.26002

[18] G. Peano, Sulla formula di Taylor. Torino Atti XXVII. 40-46. (1891). Zbl 23.0253.01

[19] J.-P. Rolin, P. Speissegger, A. J. Wilkie, Quasianalytic Denjoy-Carleman classes and o-minimality. J. Amer. Math. Soc. 16 (2003), 751-777. MR1992825 (2004g:14065) Zbl 1095.26018

[20] J.-C. Tougeron, Idéaux de fonctions différentiables. Springer 1972. MR0440598 (55 \#13472) Zbl 0251.58001

[21] L. van den Dries, Tame topology and o-minimal structures. Cambridge Univ. Press 1998. MR1633348 (99j:03001) Zbl 0953.03045 
[22] L. van den Dries, C. Miller, On the real exponential field with restricted analytic functions. Israel J. Math. 85 (1994), 19-56. MR1264338 (95e:03099) Zbl 0823.03017

[23] L. van den Dries, C. Miller, Geometric categories and o-minimal structures. Duke Math. J. 84 (1996), 497-540. MR1404337 (97i:32008) Zbl 0889.03025

[24] L. van den Dries, P. Speissegger, The real field with convergent generalized power series. Trans. Amer. Math. Soc. 350 (1998), 4377-4421. MR1458313 (99a:03036) Zbl 0905.03022

[25] L. van den Dries, P. Speissegger, The field of reals with multisummable series and the exponential function. Proc. London Math. Soc. (3) 81 (2000), 513-565. MR1781147 (2002k:03057) Zbl 1062.03029

[26] H. Volkmer, Extending Peano derivatives: necessary and sufficient conditions. Fund. Math. 159 (1999), 219-229. MR1680638 (2000b:26009) Zbl 0945.26008

[27] H. Whitney, Analytic extensions of differentiable functions defined in closed sets. Trans. Amer. Math. Soc. 36 (1934), 63-89. MR1501735 Zbl 0008.24902

[28] A. J. Wilkie, A theorem of the complement and some new o-minimal structures. Selecta Math. (N.S.) 5 (1999), 397-421. MR1740677 (2001c:03071) Zbl 0948.03037

Received 26 September, 2007

A. Fischer, Department of Mathematics \& Statistics, University of Saskatchewan, 106 Wiggins Road, Saskatoon, SK, S7N 5E6, Canada Current address: Fields Institute, 222 College Street, Toronto, Ontario, M5T 3J1, Canada Email: el.fischerandreas@web.de 